\title{
OPEN N-doped graphene foam obtained by microwave-assisted exfoliation of graphite
}

\author{
Malgorzata Skorupska ${ }^{1}$, Anna Ilnicka ${ }^{1 凶}$ \& Jerzy P. Lukaszewicz ${ }^{1,2}$
}

The synthesis of metal-free but electrochemically active electrode materials, which could be an important contributor to environmental protection, is the key motivation for this research approach. The progress of graphene material science in recent decades has contributed to the further development of nanotechnology and material engineering. Due to the unique properties of graphene materials, they have found many practical applications: among others, as catalysts in metal-air batteries, supercapacitors, or fuel cells. In order to create an economical and efficient material for energy production and storage applications, researchers focused on the introduction of additional heteroatoms to the graphene structure. As solutions for functionalizing pristine graphene structures are very difficult to implement, this article presents a facile method of preparing nitrogendoped graphene foam in a microwave reactor. The influence of solvent type and microwave reactor holding time was investigated. To characterize the elemental content and structural properties of the obtained $\mathrm{N}$-doped graphene materials, methods such as elemental analysis, high-resolution transmission electron microscopy, scanning electron microscopy, and Raman spectroscopy were used. Electrochemical activity in ORR of the obtained materials was tested using cyclic voltamperometry (CV) and linear sweep voltamperometry (LSV). The tests proved the materials' high activity towards ORR, with the number of electrons reaching 3.46 for tested non-Pt materials, while the analogous value for the C-Pt (20 wt\% loading) reference was 4.

Graphene possesses excellent mechanical and thermal properties ${ }^{1-3}$ and a high theoretical specific surface area $\left(2630 \mathrm{~m}^{2} \mathrm{~g}^{-1}\right)^{4}$. Therefore, over the past decades, graphene's potential uses have been examined within many fields ${ }^{5}$ : fields emission (FE) devices ${ }^{6}$, sensors and biosensors ${ }^{7}$, optoelectronic devices (transparent electrodes for solar cells), liquid-crystal displays (LCD) ${ }^{8,9}$, and electrode materials in batteries and supercapacitors ${ }^{10,11}$. Graphene can exist in the form of zero-dimensional (0D) dots (nanospheres ${ }^{12}$, hollow spheres ${ }^{13}$ ), one-dimensional (1D) fibers (ribbons) ${ }^{14}$, two-dimensional (2D) sheets ${ }^{15}$, and three-dimensional (3D) foams ${ }^{16}$. 3D graphene foam is a network of connected graphene sheets and, just as 2D graphene, can be widely used to improve the electrical, mechanical, or thermal properties of electrochemical devices. The size, quality, and type of graphene sheet synthesis determines the properties and potential application of the $3 \mathrm{D}$ materials ${ }^{17}$. The synthesis of a threedimensional graphene structure is based on either the assembly of two-dimensional graphene flakes or on a direct synthesis. Many papers describe 3D structure synthesis based on chemical vapor deposition (CVD) of graphene on a nickel foam matrix ${ }^{18-21}$. A relatively new synthesis method is the $3 \mathrm{D}$ printing of graphene materials ${ }^{22-24}$. Well-known methods of synthesis produce three-dimensional graphene materials effectively, but they are too expensive and require specialized equipment. The assembly of graphene flakes ${ }^{25,26}$ by the hydrothermal $\operatorname{method}^{27}$ is also popular, although not without disadvantages; self-organization of graphene sheets is the most common problem that researchers want to eliminate ${ }^{28}$. The microwave method ${ }^{29,30}$ supports the synthesis of graphene, combining many advantages: it is fast, simple, does not require specialized equipment, and the starting material for foam production can be expanded graphite ${ }^{31}$ or sucrose $^{32}$.

Graphene foams, due to their excellent porous structure, are a light material that combines the properties of two-dimensional graphene sheets and the three-dimensional system they form when joined together. The large pore volume and subsequent high specific surface area of the material, its thermal stability, high electronic conductivity, and high ion transfer rate all contribute to graphene foams' functionality in energy storage devices ${ }^{28,33,34}$. Hence, to further improve their properties, increase electrode capacity, and power or energy density, many researchers try to dope graphene materials with metal compounds or metal oxides, or to create composites with

${ }^{1}$ Faculty of Chemistry, Nicolaus Copernicus University in Torun, Gagarina 7, 87-100 Torun, Poland. ${ }^{2}$ Centre for Modern Interdisciplinary Technologies, Nicolaus Copernicus University in Torun, Wilenska 4, 87-100 Torun, Poland. ${ }^{\boxplus}$ email: ailnicka@umk.pl 


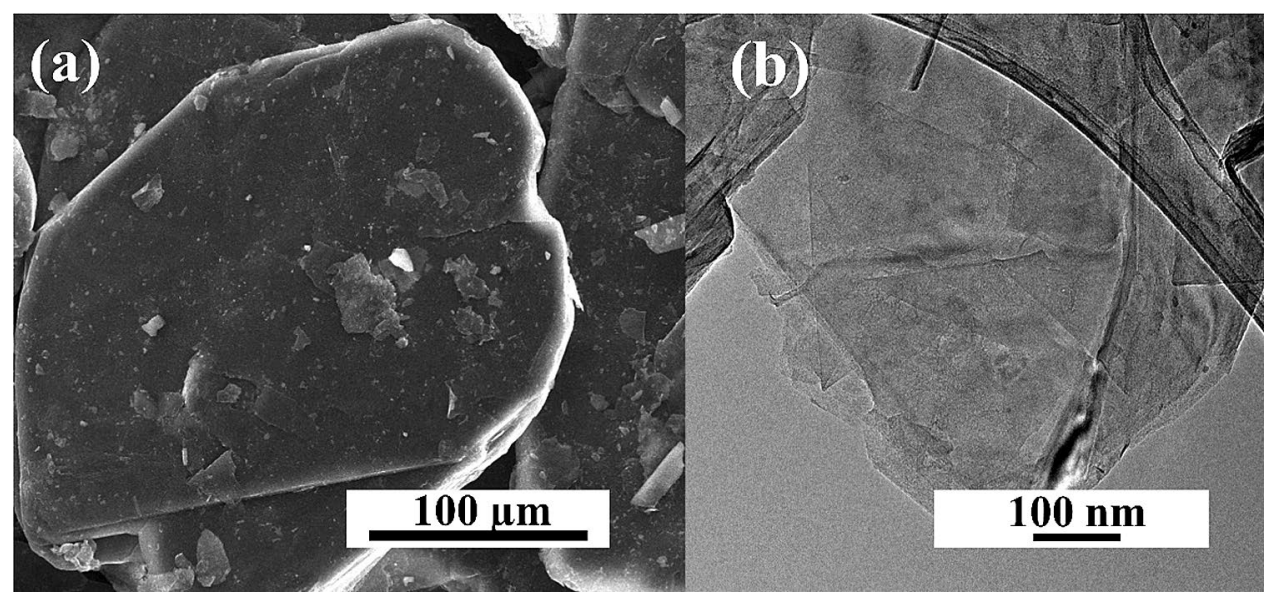

Figure 1. (a) SEM and (b) HRTEM images of expanded graphite.

other active materials ${ }^{35-38}$. These solutions involve increased production and disposal costs. The fact that these substances are not environmentally friendly leads researchers to try to introduce heteroatoms (mainly nitrogen) into the carbon structure using natural reagents ${ }^{39,40}$, thus increasing the range of potential applications of 3D graphene materials.

The synthesis of any metal-free but electrochemically active electrode material is an important contribution to protecting the environment and the key motivation for engaging this research approach. To this end, we present a fast and facile method of producing $\mathrm{N}$-doped graphene foam using a microwave process. In the proposed process, expanded graphite was used as a starting material and green algae Chlorella vulgaris as an $\mathrm{N}$-precursor. Different solvents (ethyl alcohol and dimethylformamide) were applied in the microwave reactor study. After the microwave process, the samples were carbonized. The study investigated the influence that the microwave (duration time) and carbonization processes had on graphene structure and nitrogen content.

\section{Results and discussion}

Material characterization. The proposed method to obtain N-doped graphene foam using a pressure microwave reactor was confirmed to be effective using instrumental methods. Figure 1 shows the commercial expanded graphite, the substrate for synthesis. The morphology of expanded graphite is flat and graphite sheets overlap in stacks. The scanning electron microscope images allow determining the impact of the microwave process and type of solvent on the structure of the obtained graphene materials. Figure 2 shows the structure of materials obtained in the EtOH solution for samples $0 \mathrm{~A}-10$ and $1 \mathrm{~A}-10$. For material obtained without the carbonization process, the structure is similar to pristine graphite with the layers not separated (Fig. 2a). The HRTEM images in Fig. $2 b$ indicate that the material consisted of many graphene sheets forming thick stacked layers. Figure 2c,d show SEM and HRTEM images of graphene foams obtained with N-reagent. The carbonization process aided the exfoliation of graphene flakes. The SEM and HRTEM images for samples obtained in DMF are presented in Fig. 3a,b for the 0B-10 sample and Fig. 3c,d for the 1B-10 sample. The morphology of the materials obtained in DMF is very similar to that of materials obtained in $\mathrm{EtOH}$, so further analysis was necessary. In all solvents, the material was exfoliated after carbonization to form graphene foam, creating very light and fluffy structures. The high temperature caused the creation of spaces and a system of very thin graphene walls which incorporated nitrogen atoms. The HRTEM images for all solvents indicate that in both cases very thin parallel layers can be observed. Disordered morphology and clearly overlapping layers are typical for graphene-based materials.

A basic premise of this method of enriching the final material with nitrogen is the use of green algae. After carbonization, it can join graphene flakes (single layer graphene, few layer graphene and multilayer graphene type) into a durable, porous 3D structure resembling a foam or sponge. Aside from providing amorphous carbon matter adhering graphene flakes, green algae is a source of nitrogen. It was selected due to its useful features, such as ease of mixing with graphene flakes in $\mathrm{EtOH}$ or DMF and thermal degradation to a non-porous nitrogenrich matrix. A low carbon matrix yield from the starting green algae mass makes it possible to avoid soaking the graphene flakes and retains the graphene flakes' surface accessibility to chemical reagents, e.g., electrolytes. The green algae-originated carbon phase is assumed to be present in the form N-rich bridges between graphene flakes which enable electron transfer throughout the whole mass of material. The effective exfoliation of materials obtained with a holding time of $10 \mathrm{~min}$ in the reactor led to the investigation of the effect that extending holding time had on the structure and elemental composition of graphene foams. The influence on the nitrogen content was examined in two different electrolytes, while the carbonization process was used to change the structure of graphene by creating exfoliated graphene foam with embedded nitrogen functional groups. The elemental analysis was used to determine the composition of the $\mathrm{N}$-doped graphene foams. The influence of the elemental composition for materials in series $0 \mathrm{~A} / \mathrm{B}-\mathrm{T}$ and $1 \mathrm{~A} / \mathrm{B}-\mathrm{T}$ obtained in different solvents is shown in Table 1 . The results for samples obtained in these two series indicate that solvent and holding time in the microwave reactor influence the elemental content of the obtained materials. A high carbon content in the range from 76.06 to 95.52 


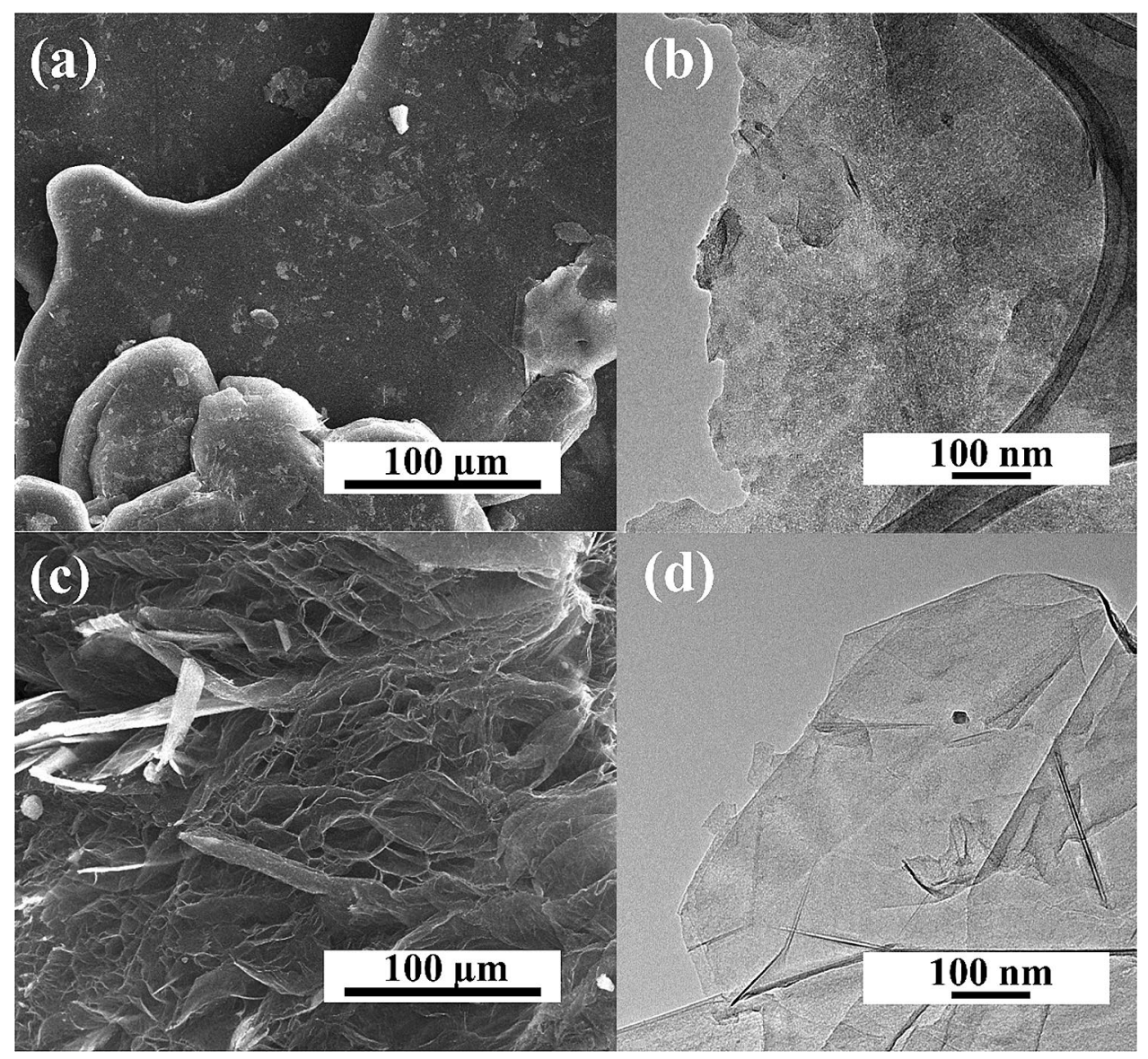

Figure 2. SEM and HRTEM images of graphene foams obtained without and with N-reagent: (a,b) $0 \mathrm{~A}-10$ and (c,d) 1A-10.

wt $\%$ was observed for all samples. The mass content of the samples obtained was typical for graphene-based materials. The nitrogen content in samples prepared without N-reagent was significantly lower than that for samples with Chlorella vulgaris. Heteroatoms were successfully introduced into the graphene structure using the natural reagent. For samples synthesized in different solvents with $\mathrm{N}$-reagent, the percentage by mass of nitrogen was high, at $2.44 \mathrm{wt} \%$ for the $1 \mathrm{~A}-10$ sample. Along with extending the reaction time for the $1 \mathrm{~A}-\mathrm{T}$ series produced in the ethyl alcohol solvent, a significant decrease of the nitrogen content, down to $0.65 \mathrm{wt} \%$, was noted. In the case of the 1B-T series, where DMF was the solvent, an increase in the samples' nitrogen content was observed when holding time was increased. The highest nitrogen content for the $1 \mathrm{~B}-\mathrm{T}$ series was recorded for a sample maintained for $90 \mathrm{~min}$, at $0.89 \mathrm{wt} \%$.

Raman spectroscopy is the method most commonly used to characterize graphene materials ${ }^{41}$. On the Raman spectra for graphene, three characteristic bands can be observed, D, G, and 2D, for which the shift at the $532 \mathrm{~nm}$ laser line is $1347 \mathrm{~cm}^{-1}, 1577 \mathrm{~cm}^{-1}$, and $2698 \mathrm{~cm}^{-142}$ respectively. Using Raman spectroscopy analysis, it is possible to estimate the number of graphene sheet layers that have been laid, as well as determine defects in the material obtained. Raman spectra were compiled for the N-doped graphene foams carried out in two selected solvents, EtOH (Fig. 4a) and DMF (Fig. 4b). The holding time in the reactor was 10, 30, 60, or 90 min. Table 2 shows the ratio of intensities of the materials obtained depending on their different reaction times and solvents. The ratio of $\mathrm{D}$ and $\mathrm{G}$ band intensities indicates the degree of graphitization defect, while $\mathrm{I}_{2 \mathrm{D}} / \mathrm{I}_{\mathrm{G}}$ indicates the number of superimposed graphene layers. Comparing the spectra with each other, there is no significant difference in the intensity ratio of $2 \mathrm{D}$ to $\mathrm{G}$ bands. The ratio is close to 0.5 , which indicates that the obtained material possesses several layers. The change in D-band is clearly visible, which may mark the quality of the graphene structure. In the $1 \mathrm{~A}$ - $\mathrm{T}$ series, the $\mathrm{I}_{\mathrm{D}} / \mathrm{I}_{\mathrm{G}}$ band ratio increases along with the reaction time, indicating quality. The low ratio of $\mathrm{D}$ to $\mathrm{G}-\mathrm{band}$ intensities suggests that graphene material was effectively obtained. These can potentially be used in electrochemical applications as electrodes, as sensors, or biosensors.

In order to determine the thickness and lateral size of graphene sheets which were treated in the microwave reactor, AFM analysis was performed using the $1 \mathrm{~A}-\mathrm{T}$ and $1 \mathrm{~B}-\mathrm{T}$ series of samples, synthesized in two solvents, EtOH (Fig. 5a) and DMF (Fig. 5b). Knowing the thickness of single-layered graphene and the interlayer distance, the measured value corresponds to few graphene layers. The thickness of graphene sheets for series $1 \mathrm{~A}-\mathrm{T}$ and $1 \mathrm{~B}-\mathrm{T}$ is in the range of $3.62-6.78 \mathrm{~nm}$ and $5.59-16.45 \mathrm{~nm}$, respectively. This result is consistent with Raman spectroscopy examinations, which also suggests that employing the microwave reactor produces few-layered graphene, regardless of the type of solvent. 


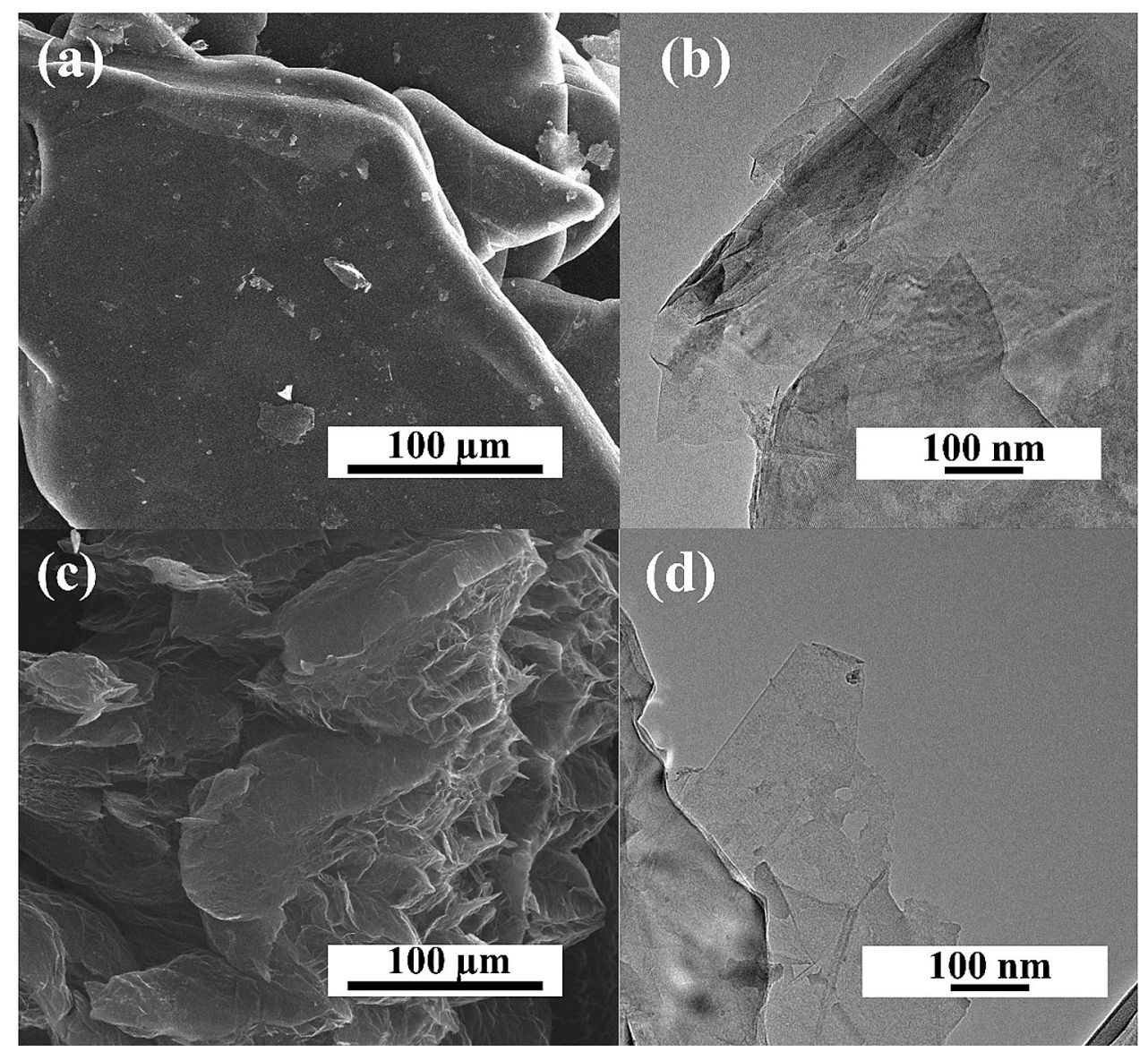

Figure 3. SEM, HRTEM images of graphene foams obtained without and with N-reagent: (a,b) 0B-10 and (c,d) $1 \mathrm{~B}-10$.

\begin{tabular}{|l|l|l|l|}
\hline \multirow{2}{*}{ Sample } & \multicolumn{3}{|l|}{ Content (wt\%) } \\
\cline { 2 - 4 } & $\mathbf{C}$ & $\mathbf{H}$ & $\mathbf{N}$ \\
\hline EG & 73.54 & 1.50 & 0.08 \\
\hline 0A-10 & 76.06 & 1.56 & 0.14 \\
\hline 0B-10 & 80.70 & 1.37 & 0.58 \\
\hline 1A-10 & 88.83 & 0.58 & 2.44 \\
\hline 1A-30 & 90.40 & 0.61 & 0.97 \\
\hline 1A-60 & 91.09 & 0.90 & 0.65 \\
\hline 1A-90 & 91.50 & 0.83 & 0.73 \\
\hline 1B-10 & 95.48 & 0.34 & 0.10 \\
\hline 1B-30 & 91.27 & 0.72 & 0.76 \\
\hline 1B-60 & 89.21 & 0.92 & 0.84 \\
\hline 1B-90 & 89.78 & 0.83 & 0.89 \\
\hline
\end{tabular}

Table 1. Elemental composition of EG and graphene foams obtained with different solvents and holding times in the microwave reactor.

The surface elemental composition of materials from the 1A-T series was analyzed using X-ray photoelectron spectroscopy. Figure 6a shows a wide survey scan for sample 1A-30 collected from 0 to $1300 \mathrm{eV}$. XPS spectra of sample 1A-30 for C1s, N1s, and O1s are presented in Fig. 6b-d. The high-resolution spectrum of C1s (Fig. 6b) can be deconvoluted into five peaks centered at $284.6 \mathrm{eV}, 285.0 \mathrm{eV}, 286.4 \mathrm{eV}, 287.7 \mathrm{eV}$, and $288.6 \mathrm{eV}$, which can be attributed to bonds of $\mathrm{C}=\mathrm{C}\left(\mathrm{sp}^{2}\right), \mathrm{C}-\mathrm{C}\left(\mathrm{sp}^{3}\right), \mathrm{C}-\mathrm{O}-\mathrm{C}, \mathrm{C}=\mathrm{O}$, and $\mathrm{O}-\mathrm{C}=\mathrm{O}$, respectively. The total content of carbon for all samples, determined by XPS measurement, was in the range from 90.2 to 93.2 at.\% (Table 3) and was at the same level as carbon content determined by elemental combustion measurement.

After deconvolution of the high-resolution spectrum of N1s, two characteristic types of bonds were determined for nitrogen in the form of pyrrolic- $\mathrm{N}$ and quaternary- $\mathrm{N}$ in binding energies of $399.1 \mathrm{eV}$ and $400.8 \mathrm{eV}$, 
(a)

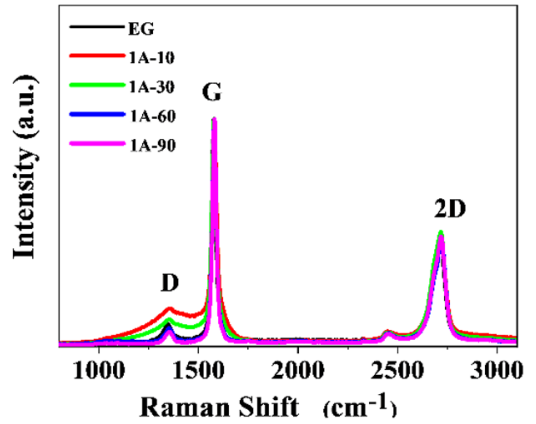

(b)

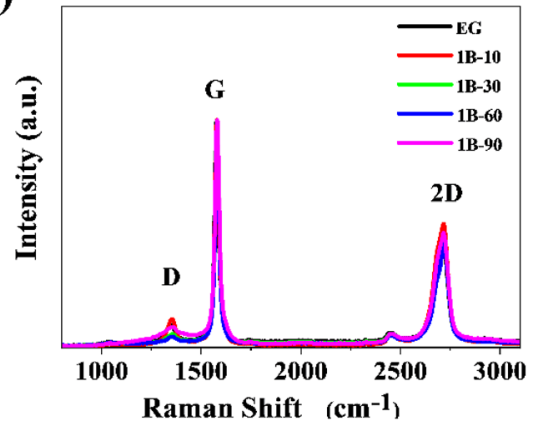

Figure 4. Raman spectra of (a) $1 \mathrm{~A}-\mathrm{T}$ and (b) $1 \mathrm{~B}-\mathrm{T}$ series compared to pristine EG.

\begin{tabular}{|l|l|l|l|l|l|l|l|l|}
\hline \multirow{2}{*}{ Sample } & \multicolumn{2}{l|}{ D-band } & \multicolumn{2}{l|}{ G-band } & \multicolumn{2}{l|}{ 2D-band } & & \\
\cline { 2 - 8 } & $\mathbf{c m}^{-1}$ & $\mathbf{I}$ & $\mathbf{c m}^{-1}$ & $\mathbf{I}$ & $\mathbf{c m}^{-1}$ & $\mathbf{I}$ & $\mathbf{I}_{\mathbf{D}} / \mathbf{I}_{\mathbf{G}}$ & $\mathbf{I}_{\mathbf{2 D}} / \mathbf{I}_{\mathbf{G}}$ \\
\hline EG & 1348.0 & 0.10 & 1575.5 & 1 & 2713.5 & 0.48 & 0.10 & 0.48 \\
\hline 1A-10 & 1353.0 & 0.17 & 1580.0 & 1 & 2716.0 & 0.47 & 0.17 & 0.47 \\
\hline 1A-30 & 1353.0 & 0.12 & 1578.5 & 1 & 2716.0 & 0.50 & 0.12 & 0.50 \\
\hline 1A-60 & 1352.5 & 0.08 & 1580.0 & 1 & 2718.5 & 0.48 & 0.08 & 0.48 \\
\hline 1A-90 & 1353.0 & 0.07 & 1580.0 & 1 & 2717.5 & 0.48 & 0.07 & 0.48 \\
\hline 1B-10 & 1353.0 & 0.12 & 1578.5 & 1 & 2716.0 & 0.55 & 0.12 & 0.55 \\
\hline 1B-30 & 1355.5 & 0.06 & 1580.0 & 1 & 2717.5 & 0.49 & 0.06 & 0.49 \\
\hline 1B-60 & 1353.0 & 0.05 & 1580.0 & 1 & 2719.0 & 0.47 & 0.05 & 0.47 \\
\hline 1B-90 & 1356.5 & 0.09 & 1580.0 & 1 & 2715.5 & 0.50 & 0.09 & 0.50 \\
\hline
\end{tabular}

Table 2. Raman band positions and intensities, as well as the intensity ratio of EG and graphene obtained in $1 \mathrm{~A}-\mathrm{T}$ and $1 \mathrm{~B}-\mathrm{T}$ series.

(a)

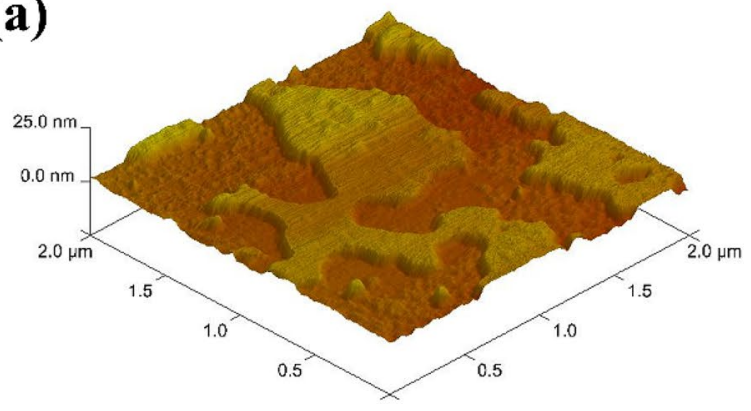

(b)

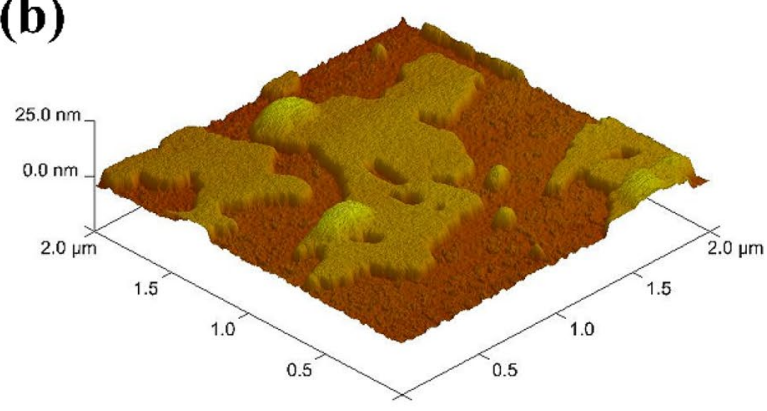

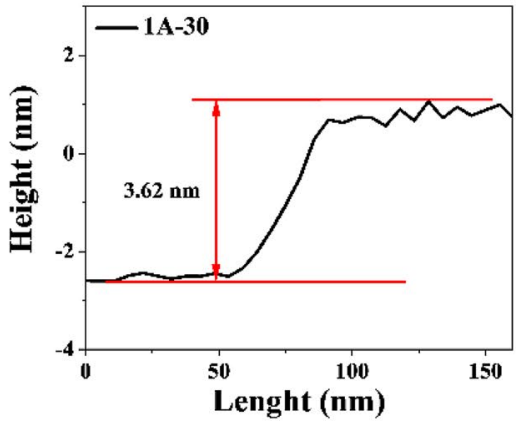
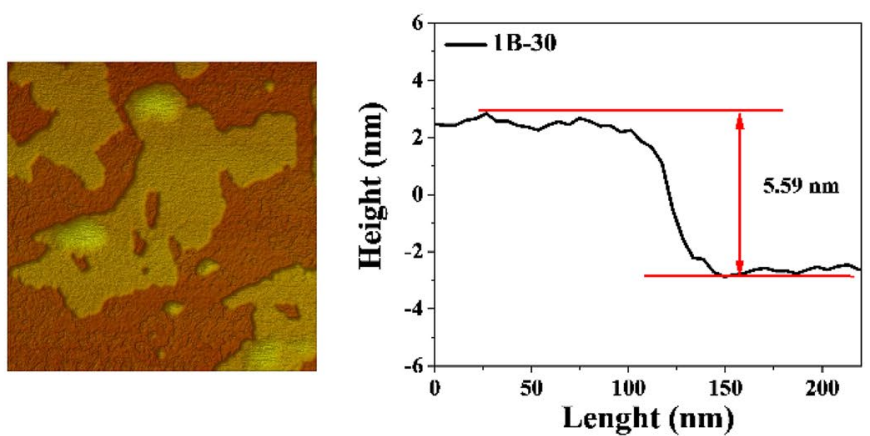

Figure 5. AFM images of (a) 1A-30, (b) 1B-30 with height profiles of graphene flakes. 
(a)

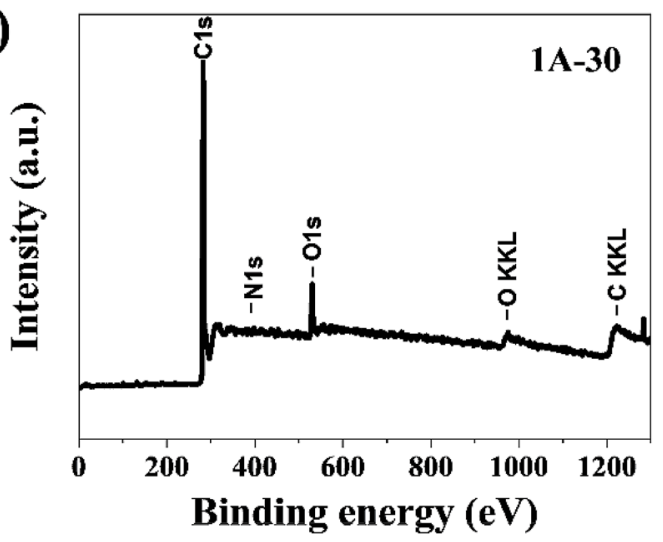

(c)

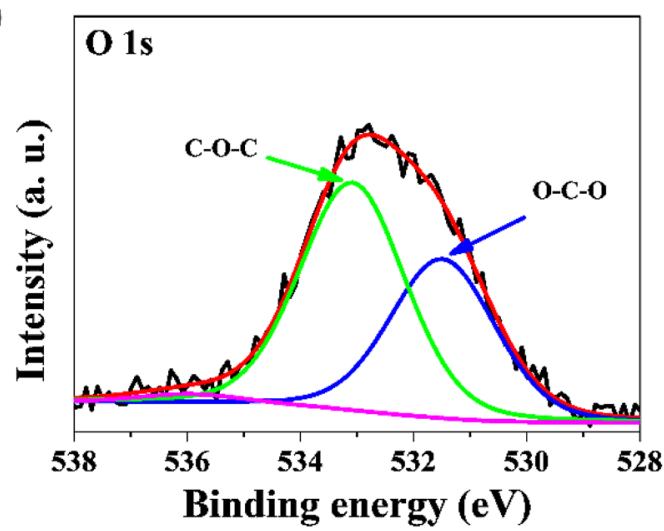

(b)

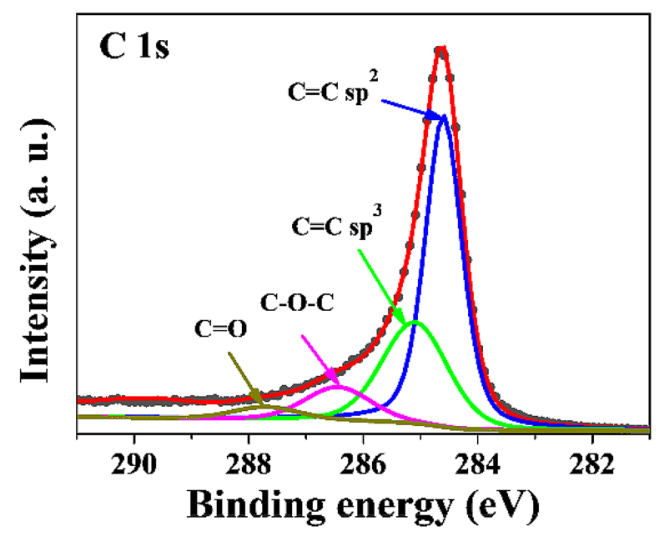

(d)

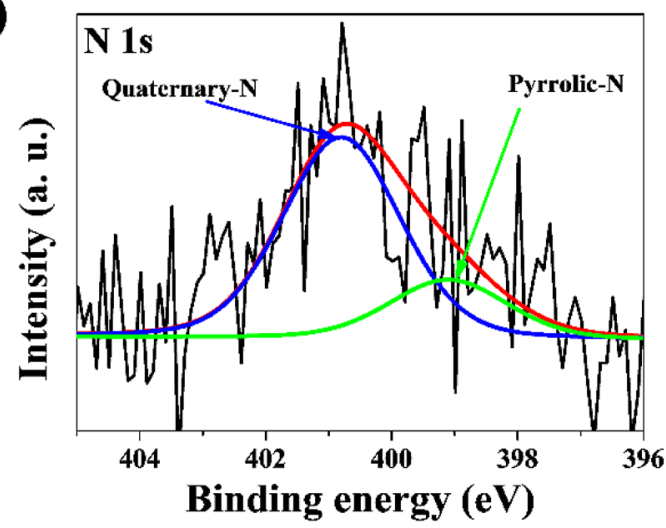

Figure 6. (a) XPS survey spectrum and high-resolution XPS spectra of (b) C1s, (c) O1s, (d) N1s for 1A-30 sample.

\begin{tabular}{|c|c|c|c|c|c|c|c|c|c|c|}
\hline \multirow[b]{4}{*}{ Sample } & \multicolumn{10}{|c|}{ Binding energy (eV) } \\
\hline & 284.6 & 285.0 & 286.4 & 287.7 & 288.6 & 531.6 & 533.2 & 536.0 & 399.1 & 400.8 \\
\hline & \multicolumn{10}{|c|}{ Elemental content (at.\%) } \\
\hline & \multicolumn{5}{|l|}{ C } & \multicolumn{3}{|l|}{$\mathbf{O}$} & \multicolumn{2}{|l|}{$\mathbf{N}$} \\
\hline $1 \mathrm{~A}-10$ & 35.7 & 41.3 & 9.3 & 3.0 & 0.9 & 2.7 & 4.5 & 0.2 & 0.9 & 0.8 \\
\hline $1 \mathrm{~A}-30$ & 57.6 & 24.0 & 7.9 & 2.9 & 0.0 & 2.5 & 3.5 & 0.1 & 0.7 & 0.2 \\
\hline $1 \mathrm{~A}-60$ & 66.5 & 16.0 & 7.9 & 2.6 & 0.0 & 2.4 & 3.2 & 0.1 & 0.5 & 0.3 \\
\hline $1 \mathrm{~A}-90$ & 59.9 & 23.5 & 7.1 & 2.7 & 0.0 & 2.4 & 2.9 & 0.3 & 0.4 & 0.2 \\
\hline
\end{tabular}

Table 3. Chemical composition analyzed using high-resolution XPS for series $1 \mathrm{~A}-\mathrm{T}$.

respectively ${ }^{43}$. Modified graphene structures subjected to microwave heating facilitated nitrogen substitution in the graphite plane. The effect of high carbonization temperatures on doped structures was the formation of mainly quaternary- $\mathrm{N}$ functional groups, responsible for the materials' effective catalytic activity in the oxygen reduction reaction ${ }^{44}$. Due to access to an electron pair in the graphene structure, the pyrolytic N-group is active in response to the oxygen reduction reaction, which leads to an increase in the catalytic activity of $\mathrm{N}$-doped materials ${ }^{45}$. The overall content of nitrogen atoms for all samples in the 1A-T series was between 0.6 and 2.7 at.\%. The XPS results show that nitrogen doping in graphene is successful; there were two characteristic types of nitrogen functional groups that improved catalytic properties in response to ORR results, the highest content belonging to the pyrrolic-N groups. Deconvolution of the high-resolution O1s spectrum fit into three bands at energy values of $531.6 \mathrm{eV}, 533.2 \mathrm{eV}$, and $536.0 \mathrm{eV}$ which correspond to $\mathrm{C}-\mathrm{O}-\mathrm{C}, \mathrm{O}-\mathrm{C}-\mathrm{O}$, and absorbed $\mathrm{H}_{2} \mathrm{O}$ bonds, respectively ${ }^{46}$.

Electrochemical performance. In order to check the catalytic activity of the obtained materials in the oxygen reduction reaction, cyclic voltammetry and linear voltammetry measurements were taken. The ORR reaction is distinctive in metal-air batteries and fuel cells, therefore examining the catalytic activity of the produced nitrogen-doped materials was an important aspect of the work. In recent years, heteroatom-doped graphene has become an alternative to platinum-based materials owing to the heteroatoms' improvement of electrochemi- 
(a)

(c)
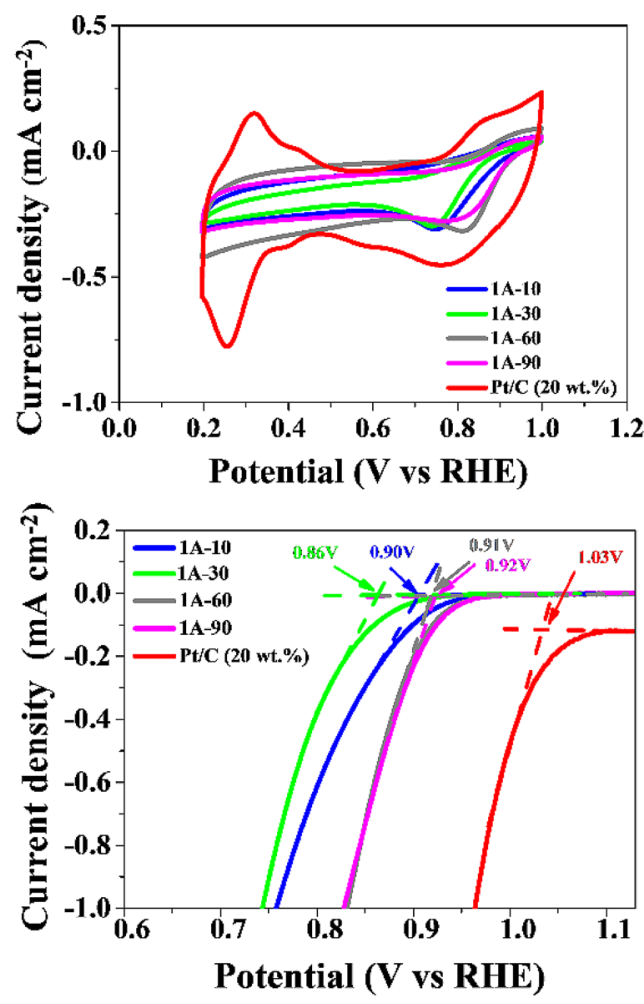

(b)

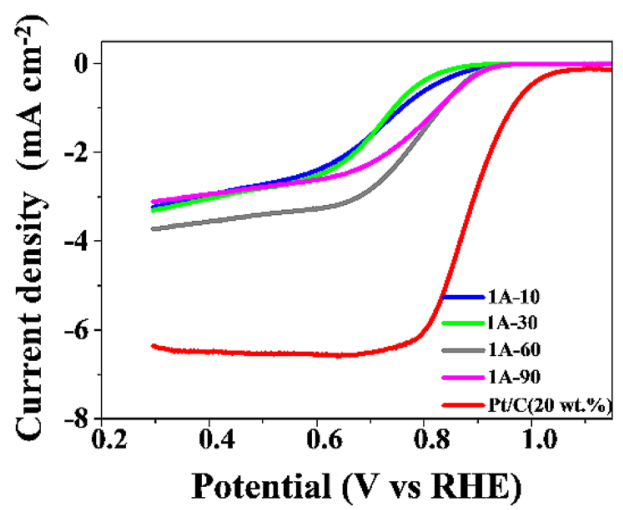

(d)

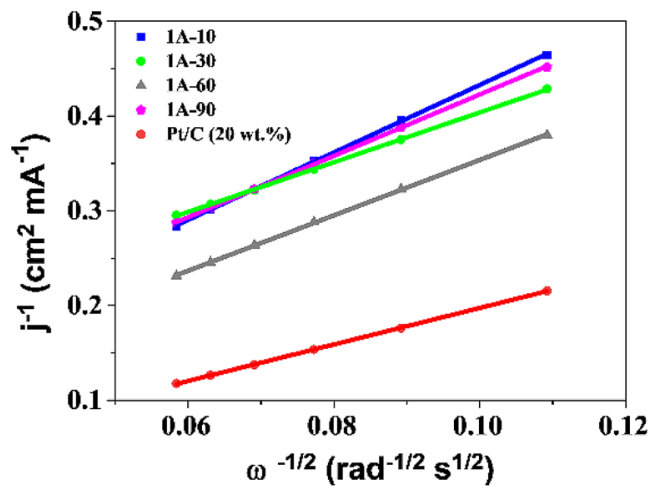

Figure 7. (a) $\mathrm{CV}$ curves with a scan speed of $100 \mathrm{mV} \mathrm{s}^{-1}$ in $\mathrm{O}_{2}$-saturated electrolyte for the $1 \mathrm{~A}$ - $\mathrm{T}$ series and the Pt/C catalyst; (b) LSV curve of the obtained samples and Pt/C catalysts (5 V s${ }^{-1}, 1600 \mathrm{rpm}$ ); (c) Onset potential of 1A-T and the commercial catalyst; (d) Koutecky-Levich plot for the samples at $0.45 \mathrm{~V}$.

cal properties ${ }^{47,48}$. In this case, graphene structures served as a matrix for the introduction of nitrogen, using the natural substrate Chlorella vurgalis as the source. As the literature reports, N-precursor is transformed into conductive carbon forms under the influence of high carbonization temperatures, $800-900{ }^{\circ} \mathrm{C}$, which increase electrocatalytic properties ${ }^{49,50}$. The reference material in electrochemical tests was a carbon material based on platinum $(20 \mathrm{wt} \%)$. All measurements were made in an oxygen-saturated $0.1 \mathrm{M} \mathrm{KOH}$ electrolyte for the materials from the $1 \mathrm{~A}-\mathrm{T}$ and $1 \mathrm{~B}-\mathrm{T}$ series, however the plots are presented for the $1 \mathrm{~A}-\mathrm{T}$ series only (Fig. 7). Figure 7a illustrates the $\mathrm{CV}$ curves for the samples obtained in the $1 \mathrm{~A}-\mathrm{T}$ series, showing the cathode peaks for samples $1 \mathrm{~A}-10,1 \mathrm{~A}-30,1 \mathrm{~A}-60$, and $1 \mathrm{~A}-90$ at a potential of $0.75 \mathrm{~V}, 0.74 \mathrm{~V}, 0.81 \mathrm{~V}$, and $0.76 \mathrm{~V}$ relative to the RHE electrode, respectively. This is one proof that the presented method produces materials with good electrocatalytic properties. When examining the catalytic activity in the oxygen reduction reaction, one should keep in mind the measurements of linear voltammetry, which provide the most information regarding the catalytic properties of the obtained materials. Figure $7 \mathrm{~b}$ shows LSV measurements. The curves were retrieved with oxygen flow at a scanning speed of $5 \mathrm{mV} \mathrm{s}$, and rotation speed of $800-2800 \mathrm{rpm}$. A curve was selected for all samples in the $1 \mathrm{~A}-\mathrm{T}$ series and for the $\mathrm{Pt} / \mathrm{C}$ reference catalyst $(20 \mathrm{wt} \%)$ at one rotation speed, $1600 \mathrm{rpm}$. Sample 1A_60 exhibits the highest current density among the samples obtained, but it is still lower than a commercial material based on platinum. This proves that, despite sample 1A-60 having the lowest nitrogen content at $0.65 \mathrm{wt} \%$, the material shows good catalytic properties in ORR. The value of the onset potential for the 1A-60 sample, shown in Fig. 7c, was $0.91 \mathrm{~V}$, while for the commercial material this value was about $1.03 \mathrm{~V}$. For the other samples, $1 \mathrm{~A}-10,1 \mathrm{~A}-30$, and $1 \mathrm{~A}-90$, the value of the onset potential was $0.90,0.86$, and $0.92 \mathrm{~V}$ vs RHE, respectively. LSV results are typical for non-metallic graphene-based materials. They contributed to the estimation of the electron transfer number in the oxygen reduction reaction, which primarily describes the path of the ORR reaction. By analyzing the Koutecky-Levich plot (Fig. 7d), it is possible to determine the number of electron transfers. Data gathered from the LSV plot at a value of $0.45 \mathrm{~V}$ were used to calculate the number of electrons participating in the reaction. For the commercial material based on platinum, a four-electron reaction was a distinguishing characteristic, while graphene is characterized by a two-electron oxygen reduction reaction ${ }^{51}$. ORR performance parameters for the $1 \mathrm{~A}$-T series and commercial platinum-based carbon are presented in Table 4 . The values closest to those for the commercial catalyst were those of samples $1 \mathrm{~A}-30$ and 1A-60, of 3.46 and 3.10, respectively. A high value of $\mathrm{n}$ means that the electron transfer in the oxygen reduction reaction is close to the 4 electron ORR mechanism. The number of transferred electrons for the series of samples obtained with DMF as solvent equals 2.55, 2.42, 2.49, and 2.57 for $1 \mathrm{~B}-10,1 \mathrm{~B}-30,1 \mathrm{~B}-60$, and $1 \mathrm{~B}-90$, respectively. The produced materials show improved properties over pristine graphene, whose typical number of electron transfers in ORR is about 2 electrons ${ }^{48,52}$. The LSV results show that the resulting materials have good catalytic properties. This is proof that the electrocatalysts doped with heteroatomic nitrogen, and mainly nitrogen functional groups in the graphene structure, are responsible for catalyzing the ORR reaction. The products are completely free of any metals and can potentially achieve 


\begin{tabular}{|l|l|l|l|l|}
\hline Sample & $\mathbf{E}_{\text {onset }}(\mathbf{V}$ vs $R H E)$ & $\mathbf{E}_{\mathbf{1} / \mathbf{2}}$ (V vs RHE) & Diffusion limiting current density $\left(\mathbf{m A ~} \mathbf{c m}^{-2}\right)$ & $\mathbf{n}(\mathbf{0 . 4 5} \mathbf{V})$ \\
\hline $1 \mathrm{~A}-10$ & 0.90 & 0.73 & 2.56 & 2.55 \\
\hline $1 \mathrm{~A}-30$ & 0.86 & 0.72 & 2.44 & 3.46 \\
\hline $1 \mathrm{~A}-60$ & 0.91 & 0.79 & 3.55 & 3.10 \\
\hline $1 \mathrm{~A}-90$ & 0.92 & 0.80 & 3.12 & 2.80 \\
\hline Pt/C $(20 \mathrm{wt} \%)$ & 1.03 & 0.88 & 6.37 & 4.00 \\
\hline
\end{tabular}

Table 4. ORR performance parameters of series $1 \mathrm{~A}-\mathrm{T}$ and commercial $\mathrm{Pt} / \mathrm{C}$ catalyst tested in alkaline media.

wide application in the field of electrochemistry. Remarkably, the 1A-60 and 1A-90 exhibited prominent ORR activity with the most positive onset potential $\left(\mathrm{E}_{\text {onset }}\right)$ at $0.91 \mathrm{~V}$ and $0.92 \mathrm{~V}$. The half-way potential $\left(\mathrm{E}_{1 / 2}\right)$ was also highest for 1A-60 and 1A-90 compared to other samples. The prominent ORR activities of 1A-30 and 1A-60 in an alkaline medium may be benefitting from the synergistic effect of the structure and elemental composition. First, the unique few-layer architecture of graphene is conducive to exposing more of the active sites and facilitates the accessibility of oxygen. Second, the nitrogen heteroatoms can induce charge redistribution and facilitate the adsorption of oxygen and the consequent reduction reaction on carbon. The proposed materials' composition is their advantage, because generally structures described for ORR application tend to be decorated with expensive, precious, or environmentally-unfriendly metals or metal oxides, e.g., $\mathrm{Pd}, \mathrm{Ru}^{53}$, perovskie oxides ${ }^{54}$, $\mathrm{Fe}(\mathrm{II})^{55}, \mathrm{Fe}_{7} \mathrm{C}_{3}{ }^{56}$ or $\mathrm{Au}^{57}$.

\section{Conclusion}

In summary, an effective method of obtaining $\mathrm{N}$-doped graphene in a microwave reactor was developed, one which uses expanded graphite and Chlorella vurgalis. Research indicates that the use of a natural N-reagent during synthesis in a microwave reactor in various solvents can contribute to the incorporation of nitrogen atoms into the graphene structure at a level of several percent. It can be concluded that ethyl alcohol and dimethylformamide are good solvents which contribute to the exfoliation of graphene layers and the production of pure graphene material. The high quality of the obtained material, without defects, shows that the method is a promising way of producing heteroatom-doped graphene foams. The optimal time for synthesis in the microwave reactor was $30 \mathrm{~min}$ and introduced mainly pyrrolic-N groups descend from $\mathrm{N}$-precursor Chlorella vurgalis. The best electrochemical results were obtained for materials using ethanol as a reaction medium. The solvent also influenced the introduction of nitrogen functional groups into the graphene structure. The products showed good catalytic activity owing to the nitrogen functional groups introduced into the graphene structure, which are responsible for the catalytic properties in the oxygen reduction reaction. The tests proved their high activity towards ORR, i.e., the number of electrons $\mathrm{n}$ reached 3.46 for tested materials (no Pt added), while the analogous value for the C-Pt (20 wt\% loading) reference was only slightly higher and equal 4 . For pristine, i.e., non-N-doped graphene, the $\mathrm{n}$ value was only 2 . It has to be underlined that the progress in electrochemical performance was achieved in an environment-friendly way, not only without the use of noble metals, but also with a reduction in energy consumption. Heteroatom doping of graphene materials is an alternative to more expensive materials based on metals, an alternative that can have promising properties for electrochemical applications and a huge impact on the potential use the composite materials in supercapacitors, metal-air batteries, or fuel cells.

\section{Materials and methods}

Preparation of $\mathbf{N}$-doped graphene foam samples. The production of $\mathrm{N}$-doped graphene foams was based on a one-step process using microwave radiation in two dispersed solvents: ethyl alcohol (EtOH), and dimethylformamide (DMF). The starting materials used were expanded commercial graphite (EG, Sinograf, Torun, Poland) and the natural green algae N-precursor, Chlorella vulgaris. The compounds were mixed in a solvent with a 1:4 mass ratio of graphene to Chlorella vulgaris, then sonicated to obtain a better dispersion. The samples were loaded into a microwave reactor (Microwave 400) and heated under pressure to $180{ }^{\circ} \mathrm{C}$, with the power of about 300-600 W. The microwaving duration was either 10, 30, 60, or $90 \mathrm{~min}$; after this time, the samples were stirred. The obtained mass was then washed with distilled water to remove the solvent and dried at $120^{\circ} \mathrm{C}$ overnight. Finally, samples were carbonized at $900{ }^{\circ} \mathrm{C}$ with a heating rate of $3{ }^{\circ} \mathrm{C} \mathrm{min} \mathrm{m}^{-1}$ and then heated for $4 \mathrm{~h}$ under the flow of nitrogen (to provide an inert environment during carbonization). Reference samples were made using the same procedure, but without the addition of an $\mathrm{N}$-precursor nor the carbonization process. For all samples without the addition of Chlorella vulgaris, the process in the reactor was maintained for $10 \mathrm{~min}$. The reference samples obtained without carbonization, only the microwave process, are designated as $0 \mathrm{~A} / \mathrm{B}-\mathrm{T}$, where $\mathrm{A}$ and $\mathrm{B}$ describe the solvent and denote EtOH or DMF, respectively, while $\mathrm{T}$ is the time in minutes that the sample was held in the microwave reactor. Samples obtained with the addition of the N-precursor and using carbonization are designated as $1 \mathrm{~A} / \mathrm{B}-\mathrm{T}$, where $\mathrm{A}$ and $\mathrm{B}$ describe the solvent and mark EtOH or DMF, respectively, and $\mathrm{T}$ is the duration of time spent in the microwave.

Characterization methods. The atomic structure of the obtained materials was analyzed using a highresolution transmission electron microscope (HRTEM FEI Tecnai F20 X-Twin, Brno, Czech Republic) at an accelerating voltage of $200 \mathrm{kV}$. A scanning electron microscope operating at $30 \mathrm{kV}$ (SEM, $1430 \mathrm{VP}$, LEO Electron Microscopy Ltd., Oberkochen, Germany) was used to determine the structure of the samples. The elemental 
composition of the materials was analyzed by means of a combustion elemental analyzer (Vario MACRO CHN, Elementar Analysensysteme GmbH, Langenselbold, Germany). Raman-microscopic spectroscopy analysis was performed using Renishaw InVia (Renishaw Company, Gloucestershire, the United Kingdom), excitation wavelength $532 \mathrm{~nm}$ at an ambient temperature. Nitrogen sorption isotherms were determined through nitrogen physisorption at $77 \mathrm{~K}$ in a volumetric apparatus ASAP 2010 (Micromeritics, Norcross, GA, USA). The attachment, bonding configuration, and compositional analysis of the material was carried out by X-ray photoelectron spectroscopy measurements (XPS, PHI5000 VersaProbe II Scanning XPS Microprobe, Chigasaki, Japan). A monochromatic Al-Ka X-ray $(1486.6 \mathrm{eV})$ was used as the operating excitation source. Atomic Force Microscopy (AFM) investigations were conducted using a Scanning Probe Microscope (SPM) apparatus produced by Veeco (Digital Instrument, USA), working under ambient conditions.

Electrochemical measurements. Tests of the obtained materials' electrochemical properties were carried out using the Autolab potentiostat (PGSTAT128N, The Netherlands). The standard measuring setup was made up of three electrodes-an $\mathrm{Ag} / \mathrm{AgCl}$ electrode in $3 \mathrm{M} \mathrm{KCl}$ was the reference, a Pt electrode the counter, while the working electrode was glassy carbon (GC rotating disk electrode with a $5.0 \mathrm{~mm}$ diameter) onto which ink, with the catalyst, was applied. The catalyst ink was prepared basing on the dispersion of $2.5 \mathrm{mg}$ of the test sample in a mixture of distilled water and ethanol (ratio 1:4) and Nafion (0.5 wt\% Nafion). As a point of comparison, electrochemical studies were also performed on a commercial catalyst from Sigma Aldrich, platinum on graphitized $\mathrm{Pt} / \mathrm{C}$ carbon ( $20 \mathrm{wt} \%$ Platinum). The prepared ink was pipetted onto glassy carbon in an amount of $15.63 \mu \mathrm{l}$ and the electrode was dried afterwards. Catalyst content was about $0.4 \mathrm{mg} \mathrm{cm}^{-2}$. Before electrochemical testing, the alkaline solution was saturated with $\mathrm{O}_{2} / \mathrm{N}_{2}$ for $20 \mathrm{~min}$. The activity of the samples in an oxygen reduction reaction was evaluated by measuring cyclic voltammetry $(\mathrm{CV})$, carried out at a scanning speed of $10 \mathrm{mV} \mathrm{s}^{-1}$, and linear voltammetry (LSV), at a scanning speed of $5 \mathrm{mV} \mathrm{s}^{-1}$ and rotation speed of 800-2800 rpm, in a solution of $0.1 \mathrm{M} \mathrm{KOH}$ at room temperature. The number of electrons (n) directly participating in ORR was calculated using the Koutecky-Levich equation $(\mathrm{K}-\mathrm{L})$ :

$$
\begin{gathered}
\mathrm{J}^{-1}=\mathrm{J}_{\mathrm{L}}^{-1}+\mathrm{J}_{\mathrm{K}}^{-1}=\left(\mathrm{B} \omega^{1 / 2}\right)^{-1}+\mathrm{J}_{\mathrm{K}}^{-1} \\
\mathrm{~B}=0.62 \mathrm{nFC}_{0}\left(\mathrm{D}_{0}\right)^{2 / 3} v^{-1 / 6}
\end{gathered}
$$

where $\mathrm{J}$ is defined as the measured density, $\mathrm{J}_{\mathrm{L}}$ is the current density limiting diffusion, and $\mathrm{J}_{\mathrm{K}}$ is the kinetic current density; $\omega$ is the angular velocity of the electrode; $n$ is the number of electron transfers in the reaction; $F$ is the Faraday constant $\left(96,485 \mathrm{C} \mathrm{mol}^{-1}\right) ; \mathrm{C}_{0}$ is the concentration of dissolved oxygen $\left(1.2 \times 10^{-6} \mathrm{~mol} \mathrm{~L}^{-1}\right.$ in $\left.0.1 \mathrm{M} \mathrm{KOH}\right)$; $\mathrm{D}_{0}$ is the diffusion coefficient of dissolved oxygen $\left(1.9 \times 10^{-5} \mathrm{~cm}^{2} \mathrm{~s}^{-1}\right.$ in $\left.0.1 \mathrm{M} \mathrm{KOH}\right)$; $v$ is the kinetic viscosity of the electrolyte for $0.1 \mathrm{M} \mathrm{KOH}, 0.01 \mathrm{~cm}^{2} \mathrm{~s}^{-1}$. The slope of the K-L graph can be obtained using Koutecky-Levich Eqs. (1) and (2), thanks to which it is possible to determine the number of electron transfers in ORR.

Received: 28 August 2020; Accepted: 24 December 2020

Published online: 21 January 2021

\section{References}

1. Zhu, Y. et al. Graphene and graphene oxide: Synthesis, properties, and applications. Adv. Mater. 22, 3906-3924 (2010).

2. Lee, C., Wei, X., Kysar, J. W. \& Hone, J. Measurement of the elastic properties and intrinsic strength of monolayer graphene. Science 321, 385-388 (2008)

3. Balandin, A. A. Thermal properties of graphene and nanostructured carbon materials. Nat. Mater. 10, 569-581 (2011).

4. Bhuyan, M. S. A., Uddin, M. N., Islam, M. M., Bipasha, F. A. \& Hossain, S. S. Synthesis of graphene. Int. Nano Lett. 6, 65-83 (2016).

5. Choi, W., Lahiri, I., Seelaboyina, R. \& Kang, Y. S. Synthesis of graphene and its applications: A review. Crit. Rev. Solid State Mater. Sci. 35, 52-71 (2010).

6. Kumar, S., Duesberg, G., Pratap, R. \& Raghavan, S. Graphene field emission devices. Appl. Phys. Lett. 105, 103107 (2014).

7. Justino, C. I., Gomes, A. R., Freitas, A. C., Duarte, A. C. \& Rocha-Santos, T. A. Graphene based sensors and biosensors. TrAC, Trends Anal. Chem. 91, 53-66 (2017).

8. Xu, Y. \& Liu, J. Graphene as transparent electrodes: Fabrication and new emerging applications. Small 12, 1400-1419 (2016).

9. Kumar, A., Ganguly, P. \& Biradar, A. M. Single layer graphene: an alternative electrode material for ferroelectric liquid crystal based displays. Liq. Cryst. 45, 1620-1625 (2018).

10. Bulbula, S. T., Lu, Y., Dong, Y. \& Yang, X.-Y. Hierarchically porous graphene for batteries and supercapacitors. New J. Chem. 42, 5634-5655 (2018).

11. Dai, C. et al. Recent progress in graphene-based electrodes for flexible batteries. InfoMat (2019).

12. Fan, W. et al. Nitrogen-doped graphene hollow nanospheres as novel electrode materials for supercapacitor applications. J. Power Sources 243, 973-981 (2013).

13. Wu, L., Feng, H., Liu, M., Zhang, K. \& Li, J. Graphene-based hollow spheres as efficient electrocatalysts for oxygen reduction. Nanoscale 5, 10839-10843 (2013).

14. Sheng, L. et al. Ultra-high toughness all graphene fibers derived from synergetic effect of interconnected graphene ribbons and graphene sheets. Carbon 120, 17-22 (2017).

15. Ruiz, L., Xia, W., Meng, Z. \& Keten, S. A coarse-grained model for the mechanical behavior of multi-layer graphene. Carbon $\mathbf{8 2}$, 103-115 (2015).

16. Huang, Z., Guo, H. \& Zhang, C. Assembly of 2D graphene sheets and 3D carbon nanospheres into flexible composite electrodes for high-performance supercapacitors. Compos. Commun. 12, 117-122 (2019).

17. Shen, Z., Ye, H., Zhou, C., Kröger, M. \& Li, Y. Size of graphene sheets determines the structural and mechanical properties of 3D graphene foams. Nanotechnology 29, 104001 (2018).

18. Aslam, S. et al. Graphene oxide coated graphene foam based chemical sensor. Mater. Lett. 235, 66-70 (2019).

19. Chen, Z., Xu, C., Ma, C., Ren, W. \& Cheng, H. M. Lightweight and flexible graphene foam composites for high-performance electromagnetic interference shielding. Adv. Mater. 25, 1296-1300 (2013). 
20. He, J., Chen, Y. \& Manthiram, A. MOF-derived cobalt sulfide grown on 3D graphene foam as an efficient sulfur host for long-life lithium-sulfur batteries. IScience 4, 36-43 (2018).

21. Sha, J. et al. Preparation of three-dimensional graphene foams using powder metallurgy templates. ACS Nano 10, 1411-1416 (2016).

22. Kim, J. H. et al. 3D printing of reduced graphene oxide nanowires. Adv. Mater. 27, 157-161 (2015).

23. Sha, J. et al. Three-dimensional printed graphene foams. ACS Nano 11, 6860-6867 (2017).

24. Guo, H., Lv, R. \& Bai, S. Recent advances on 3D printing graphene-based composites. Nano Mater. Sci. 1, 101-115 (2019).

25. Zou, J. \& Kim, F. Diffusion driven layer-by-layer assembly of graphene oxide nanosheets into porous three-dimensional macrostructures. Nature communications 5, 1-9 (2014).

26. Hu, G. et al. 3D graphene-foam-reduced-graphene-oxide hybrid nested hierarchical networks for high-performance Li-S batteries. Adv. Mater. 28, 1603-1609 (2016).

27. Karuppanan, K. K. et al.3D-porous electrocatalytic foam based on Pt@ N-doped graphene for high performance and durable polymer electrolyte membrane fuel cells. Sustain. Energy Fuels 3, 996-1011 (2019).

28. Karfa, P., Majhi, K. C. \& Madhuri, R. Self-standing Substrates: Materials and Applications (eds Inamuddin, Boddula, R. \& Asiri, A. M.) 327-351 (Springer International Publishing, 2020).

29. Patra, S., Choudhary, R., Roy, E., Madhuri, R. \& Sharma, P. K. Heteroatom-doped graphene 'Idli': A green and foody approach towards development of metal free bifunctional catalyst for rechargeable zinc-air battery. Nano Energy 30, 118-129 (2016).

30. Zhang, T. et al. Ultrafast microwave reduction process for high-quality graphene foam with outstanding electromagnetic interference shielding and good adsorption capacity. FlatChem 17, 100117 (2019).

31. Lee, S.-H., Kang, D. \& Oh, I.-K. Multilayered graphene-carbon nanotube-iron oxide three-dimensional heterostructure for flexible electromagnetic interference shielding film. Carbon 111, 248-257 (2017).

32. Canencia, F. et al. Conducting macroporous carbon foams derived from microwave-generated caramel/silica gel intermediates. J. Mater. Sci. 52, 11269-11281 (2017).

33. Xiong, C. et al. The recent progress on three-dimensional porous graphene-based hybrid structure for supercapacitor. Compos. $B$ Eng. 165, 10-46 (2019).

34. Xia, X., Chao, D., Zhang, Y. Q., Shen, Z. X. \& Fan, H. J. Three-dimensional graphene and their integrated electrodes. Nano Today 9, 785-807 (2014).

35. Rakhi, R., Chen, W., Cha, D. \& Alshareef, H. N. High performance supercapacitors using metal oxide anchored graphene nanosheet electrodes. J. Mater. Chem. 21, 16197-16204 (2011).

36. Manjakkal, L., Núñez, C. G., Dang, W. \& Dahiya, R. Flexible self-charging supercapacitor based on graphene-Ag-3D graphene foam electrodes. Nano Energy 51, 604-612 (2018).

37. Garakani, M. A. et al. Heterogeneous, mesoporous $\mathrm{NiCo} 2 \mathrm{O} 4-\mathrm{MnO} 2 /$ graphene foam for asymmetric supercapacitors with ultrahigh specific energies. J. Mater. Chem. A 5, 3547-3557 (2017).

38. Qian, Y. et al. A free-standing Li4Ti5O12/graphene foam composite as anode material for Li-ion hybrid supercapacitor. Electrochim. Acta 258, 1311-1319 (2017).

39. Lin, G. et al. Three-dimensional interconnected nitrogen-doped mesoporous carbons as active electrode materials for application in electrocatalytic oxygen reduction and supercapacitors. J. Colloid Interface Sci. 527, 230-240 (2018).

40. Huang, J. et al. Extremely elastic and conductive N-doped graphene sponge for monitoring human motions. Nanoscale 11, 11591168 (2019)

41. Bokobza, L., Bruneel, J.-L. \& Couzi, M. Raman spectra of carbon-based materials (from graphite to carbon black) and of some silicone composites. J. Carbon Res. 1, 77-94 (2015).

42. Malard, L., Pimenta, M., Dresselhaus, G. \& Dresselhaus, M. Raman spectroscopy in graphene. Phys. Rep. 473, 51-87 (2009).

43. Wang, T. et al. Identifying the active site of $\mathrm{N}$-doped graphene for oxygen reduction by selective chemical modification. ACS Energy Lett. 3, 986-991 (2018)

44. Rahsepar, M., Nobakht, M. R., Kim, H. \& Pakshir, M. Facile enhancement of the active catalytic sites of N-doped graphene as a high performance metal-free electrocatalyst for oxygen reduction reaction. Appl. Surf. Sci. 447, 182-190 (2018).

45. Wu, H. et al. A graphene-based electrocatalyst co-doped with nitrogen and cobalt for oxygen reduction reaction. Int. J. Hydrogen Energy 41, 20494-20501 (2016).

46. Wei, D. et al. Synthesis of N-doped graphene by chemical vapor deposition and its electrical properties. Nano Lett. 9, 1752-1758 (2009).

47. Ouyang, W. et al. Exploring the active sites of nitrogen-doped graphene as catalysts for the oxygen reduction reaction. Int. J. Hydrogen Energy 39, 15996-16005 (2014).

48. Tao, H. et al. N-Doping of graphene oxide at low temperature for the oxygen reduction reaction. Chem. Commun. 53, 873-876 (2017).

49. Ilnicka, A., Lukaszewicz, J. P., Shimanoe, K. \& Yuasa, M. Urea treatment of nitrogen-doped carbon leads to enhanced performance for the oxygen reduction reaction. J. Mater. Res. 33, 1612-1624 (2018).

50. Ilnicka, A., Gauden, P. A., Terzyk, A. P. \& Lukaszewicz, J. P. Nano-structured carbon matrixes obtained from chitin and chitosan by a novel method. J. Nanosci. Nanotechnol. 16, 2623-2631 (2016).

51. Yasuda, S., Yu, L., Kim, J. \& Murakoshi, K. Selective nitrogen doping in graphene for oxygen reduction reactions. Chem. Commun. 49, 9627-9629 (2013).

52. Lou, F. et al. One-step electrochemical synthesis of tunable nitrogen-doped graphene. J. Mater. Chem. A 4, 1233-1243 (2016).

53. Barman, B. K., Sarkar, B. \& Nanda, K. K. Pd-coated Ru nanocrystals supported on N-doped graphene as HER and ORR electrocatalysts. Chem. Commun. 55, 13928-13931 (2019).

54. Bu, Y. et al. Synergistic interaction of perovskite oxides and N-doped graphene in versatile electrocatalyst. J. Mater. Chem. A 7, 2048-2054 (2019).

55. Komba, N. et al. Iron (II) phthalocyanine/N-doped graphene: A highly efficient non-precious metal catalyst for oxygen reduction. Int. J. Hydrogen Energy 44, 18103-18114. https://doi.org/10.1016/j.ijhydene.2019.05.032 (2019).

56. Wen, G.-L. et al. Graphene wrapped Fe7C3 nanoparticles supported on N-doped graphene nanosheets for efficient and highly methanol-tolerant oxygen reduction reaction. J. Colloid Interface Sci. 556, 352-359. https://doi.org/10.1016/j.jcis.2019.08.064 (2019).

57. Yan, Y., Yan, S., Yu, Z. \& Zou, Z. Low-work-function silver activating N-doped graphene as efficient oxygen reduction catalysts in acidic medium. ChemCatChem 11, 1033-1038. https://doi.org/10.1002/cctc.201801869 (2019).

\section{Acknowledgements}

This work was carried out as a result of the research project no 2019/35/N/ST5/02691, financed by the National Science Centre. 


\section{Author contributions}

M.S.: conceptualization, methodology, formal analysis, investigation, writing—original draft, supervision, project administration, funding acquisition, visualization. A.I.: conceptualization, methodology, formal analysis, writing_original draft, writing—review and editing. J.P.L.: conceptualization, writing-review and editing.

\section{Competing interests}

The authors declare no competing interests.

\section{Additional information}

Correspondence and requests for materials should be addressed to A.I.

Reprints and permissions information is available at www.nature.com/reprints.

Publisher's note Springer Nature remains neutral with regard to jurisdictional claims in published maps and institutional affiliations.

(c) (i) Open Access This article is licensed under a Creative Commons Attribution 4.0 International License, which permits use, sharing, adaptation, distribution and reproduction in any medium or format, as long as you give appropriate credit to the original author(s) and the source, provide a link to the Creative Commons licence, and indicate if changes were made. The images or other third party material in this article are included in the article's Creative Commons licence, unless indicated otherwise in a credit line to the material. If material is not included in the article's Creative Commons licence and your intended use is not permitted by statutory regulation or exceeds the permitted use, you will need to obtain permission directly from the copyright holder. To view a copy of this licence, visit http://creativecommons.org/licenses/by/4.0/.

(C) The Author(s) 2021 